\title{
Interacting and making personalized recommendations of places of interest to tourists
}

\author{
Fábio Fernandes ${ }^{1}$, Fernando Reinaldo Ribeiro ${ }^{1,2}$ \\ ${ }^{1}$ Informatics Department, Polytechnic Institute of Castelo Branco \\ Castelo Branco, Portugal \\ ${ }^{2}$ ALGORITMI Research Centre, University of Minho \\ Guimarães, Portugal
}

\begin{abstract}
Nowadays, applications that are developed to support tourists should go much further than simply providing information about places or recommending places or routes based on the user location. They should be able to provide users with simple mechanisms to interact with places of interest and provide them with relevant information and recommendations about new relevant places of interest or tours according to their preferences and the preferences of other tourists with similar interests. In this work we describe a system that explores information about tourists' interactions with places of interest and their opinions about each place, to recommend new places of interest, pedestrian tours and to promote products and services which are in accordance with their expectations. First experiments show that the system can help the tourists to interact with places of interest, helping them in their visits and also to promote shops and services.
\end{abstract}

Keywords: personalized recommendations, pervasive systems, recommender systems, mobile computing, ubiquitous computing, urban computing.

\section{Introduction}

The proliferation of mobile computing devices and their capabilities for communication, collaboration, mobility and interaction, using many different technologies, associated with the fact that there are more and more technologies of embedded computing in everyday objects, has created opportunities to develop new applications that can help people in their everyday life. The use of these devices to collect information about their users and their surroundings, combined with the technologies supported by these devices (e.g. Bluetooth, NFC, qr-codes, Wi-Fi, GPS, etc.) allows a wide range of interaction possibilities. These capabilities enable the development of new applications in many different areas. In the tourism area they can help to promote places, tours, commercial places, etc. and applications can go much further than simply providing information about specific places or just recommending places or routes, based on the user location. To achieve this, more than simple location, systems should be aware of the users' preferences and should provide support for the interaction with the points of interest, providing users with relevant information and making recommendations about new relevant places or tours according to the 
preferences of the user and the preferences of other people with similar interests. A tourist, when arrive to a specific place, is often interested in exploring local cultures that match his personal interests, such as visiting museums, visiting shops selling local items, sampling local cuisines or understanding local customs. For these tourists common tourism resources, like tourism boards, are not suitable. They need to be informed about places that fit their preferences and not with a collection of information which required them to look for the information they want and required them to make plans for pedestrian tours that fit their specific interests.

In this work we describe a system that explores user's individual interactions and their opinions to recommend not only pedestrian tours and relevant places of interest to visit but also shops and local businesses and thus improving both visitor's satisfaction and commercial and tourism places opportunities.

The remainder of this paper will be as follows. In section 2 we describe the main goals and challenges of this system. Section 3 presents a brief review of related work. Section 4 provides an overview of some guidelines about how to use personal interactions and opinions to support the recommendation of pedestrian tours and places of interest. In section 5 we describe the prototype architecture and its main components. In section 6 we present the developed prototype and the initial experiments and finally, in Section 7, we present some conclusions and we outline some of the future work.

\section{Goals and Challenges}

The main goal of this work is to develop an information system to help tourists in their visits and to support the promotion of the tourism resources and services by recommending them jointly with pedestrian tours and places of interest which are in accordance with the interests and expectations of the users. To this end, the system should to:

- provide simple and intuitive interaction mechanisms to interact with places of interest;

- provide information about specific places and locations;

- provide support for typified pedestrian tours (e.g. entertainment, religious, historical);

- recommend pedestrian tours and places of interest based on the interests and location of the tourist;

- recommend pedestrian tours and places of interest according to the experiences and opinions of other tourists with similar interests;

- promote tourism resources and places (e.g. services, museums, handcrafts, accommodations, restaurants, etc.) according to the interests of the place visitors and their location.

However, developing systems for recommending pedestrian tours and places of interest poses many challenges that are not usually found when developing information and recommendation systems for traditional media or online media, namely:

- how to obtain information about the interests of the tourists and their opinions about places they visit; 
- how to provide easy to use and non-intrusive mechanisms to support interaction between tourists and places;

- how to get feedback of the tourists about each place of interest;

- which information should be used and how it should influence the recommendation of each place of interest.

\section{Related work}

Several information systems that incorporate recommending and navigation features have been presented and discussed in recent years. In this section we put special attention to systems that are able to provide recommendations of places or tours considering the location and preferences of the users.

ROSE [1] is a mobile application which suggests events and locations to the user and guides him to them via public transportation. This system determines the best transport link and then accompanies the passenger throughout his journey. The recommender uses a query entered by the user to generate a list of suggestions. In [2] Wan Z. propose a personalized recommendation tourism information system. In this system, RFID tags together with RFID readers provide user identification and location. Recommendation is based on implicit feedbacks which involve user's implicit operations on short messages and web pages, user's location and purchase record. All these feedbacks reflect user's interest in certain recommendation and are used to update user profile or refine recommendation. A system to provide tourists with personalized location recommendations leveraging user-generated location contents is described in [3]. They identify locally interesting venues to be those frequently visited by local people and they use this information to support recommendations for tourists. Baltrunas et al. [4] present a context aware recommender system for points of interest. This system takes into consideration contextual information such as distance to the points of interest, travel goal, companion, time day, week day, temperature, budget, users' mood, etc. Lucchese et al. ReRex [5] present a system to generate personalized recommendations of touristic places of interest based on the knowledge mined from photo albums and Wikipedia. The system exploits a context-aware rating prediction model to generate recommendations. Recommendations are personalized according to the places previously visited by the user. Hao et al. [6] proposed a location-topic model to discover local and global topics from travelogues and characterize locations using local topics and they develop a tour destination recommendation. To make recommendation, the user needs to indicate a query and the system applies the locationtopic model to select the destination that best matches the user query.

In fact, there are several systems that provide personalized recommendations of places of interest. Some of them exploit information about the context of the user, like location, time, weather, and their preferences to make personalized recommendations. This system provides mechanisms to interact with places of interest and it combines information about user' interactions and their opinions about each place to make personalized recommendations of places of interest and pedestrian tours but also to promote product and services that are related to the interests of the tourists. 


\section{Interacting, characterizing and recommending}

Several systems are able to make personalized recommendations of places of interest in public places. Most of them make use of the users' personal mobile devices to provide the user with information about places of interest considering the user location. In many cases recommendations that are delivered to the users with different interests and expectations are similar. To improve the recommendation's accuracy, recommendations should be personalized and should be provided according to the expectations of each tourist. However, without knowing some characteristics of each place of interest and without being aware of the preferences and interests of the tourists that frequent those places it is not possible to make appropriate decisions about which place of interest or which pedestrian tour to recommend to each tourist.

\subsection{Interacting with places of interest}

A very common way to specify user' preferences is using personal mobile devices and, in these cases, there are several communication mechanisms to support them (e.g. SMS, Bluetooth, Wi-Fi, NFC, qr-codes). Using these devices users are able to send specific commands, to specify keywords denoting interest in some specific topic (e.g. SMS, Bluetooth) or to read tags in products (e.g. NFC tags, qr-codes) and, with this information, systems are able to respond accordingly to their interests and thus able to make appropriate recommendations. Using these technologies, it is possible to make associations between services and/or products and the user' interactions, or build user profiles based on user past interactions and opinions. In fact information about users' individual interests can be easily obtained through simple interactions, and just based on this information the system is able to make personalized recommendations. In public places qr-codes and NFC are simple and easy to use. They are simple to install in specific places and they are simple to read using common smartphones. The association between specific places and qr-codes or NFC tags allows to build a link between the physical place and all the information about it.

\subsection{Users preferences and places characterization}

The main idea is to recommend to tourists the places of interest that are in accordance with their expectations. To do this, the system should be aware of some information about the general interests of the tourists that visit each place. Individual tourists' interactions (when they interact with a place of interest reading the associated $q r$-code or NFC tag) and the opinions of the tourists about each place they visit are central to collect knowledge about the interests of the tourists and this knowledge can be used to support the decisions about which place is more appropriate for the interests of each tourist. Using information related to the history of tourists' past interactions, places of interest they interact to, interactions of similar users and location, the system will be able to recommend other relevant places of interest. For example, if the tourist reads a qr-code associated to a museum of tissues and tapestries and if he gave positive feedback to that place, probably he would like to visit the craft shop selling tapestries 
and embroideries in the nearby street. Additionally, it is important to have a characterization of each place of interest. This information may include: name, category, keywords, location, opening and closing times, etc.

\subsection{Recommending relevant places}

Traditionally, recommender systems deal with two types of entities: users and items. The user entity is normally a user profile that is based on either manual user input of preferences or deriving user preferences from the user's history of content consumption. The item entity is usually characterized with a set of metadata, but this information can also be extended with additional information that is inserted by users. These entities are the basis of all recommendation techniques that normally depend on extensive data about both. However, the specificities of how they are used differ between the three main recommendation techniques in use today: content-based recommendations, collaborative recommendations and hybrid approaches [7]. In content-based recommendation (e.g. [8]), the system suggests, to the user, items that best fit a specified set of criteria on users' profiles. The set of attributes that characterize each item is used to determine the appropriateness of the item for recommendation purposes and the user will be recommended items similar to the ones the user preferred in the past. On collaborative recommendations (e.g. [9]), the system makes recommendations based on the information of people with similar tastes and preferences, trying to predict the utility of the items for a particular user based on the items previously rated by other users. Because collaborative systems utilize other users' recommendations, they can deal with any kind of content and recommend any items.

Other, proposals (e.g. [10]) that combine collaborative and content-based methods to avoid certain limitations of content-based and collaborative systems were emerged. This technique allows minimize some problems that are associated to collaborative or content-based techniques and thus some authors see collaborative filtering as a complementary technology to content-based filtering.

In this work, knowing the characterization of each place of interest and the interactions made by each tourist are central features to decide which place of interest is more appropriate to recommend to each tourist. The most simple recommendation process may recommend to the tourist the places of interest that are similar (according to its category) to these ones the tourist has already interacted to or gave it positive feedback. In this case the recommendations should also take into consideration the user location and the distance from the user to each place. A more elaborated approach may include information related to other tourists that visit the same places or similar places. In this case, it is possible to infer about some places of interest that the tourist probably would like to visit. For example, the tourist likes the museum and other tourists that like the same museum also like the craft shop selling tapestries. Thus, probably the tourist would like to visit the same craft shop. In both cases, recommendation of places and recommendation of tours, the system should recommend several places of interest so that the tourist may select the places that best fit his interests and ignore those who do not intend to visit. 


\section{Architecture and technologies}

Fig. 1 shows the architecture of the system that implements the proposed approach. It includes four main components: web portal, content server, places of interest and a mobile application that provides users with information and recommendations and provides support to interact with places of interest.

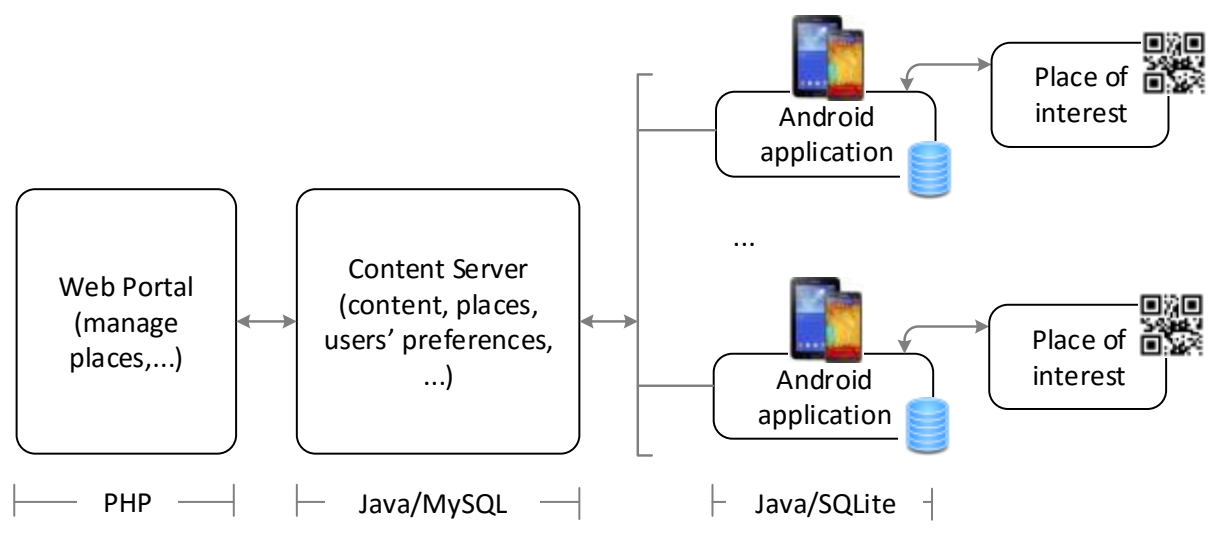

Fig. 1. System architecture and technologies.

Web Portal: the web portal is the back-office that allows to manage and to characterize the places of interest. It provides support to manage places namely, insert new places of interest, characterize them and associate each one to a qr-code. The information associated to each place of interest includes: name of the place, address, location, main category, description, image for the mobile phone, opening and closing times and a set of keywords that characterize the place.

Content server: the content server contains information associated to the places of interest and the opinions of the users on each place. It is also responsible for providing information to the android applications. This includes information about places and recommendations of new places of interest. The communication between the content server and the android application is realized using web services. Data is stored in a MySQL database.

Place of interest: a qr-code is associated to each place of interest. It acts as a link between the place of interest and the related content in the content server. When a user reads a $q$-code in a place of interest the system provides him with information related to that place of interest.

Mobile phone (android application): this application is responsible for reading the $q r$-code and it communicates with the content server to obtain information about each place and recommendations about relevant places of interest that are associated to the user location. When a user reads a qr-code in a place of interest the system provides him with information related to that place of interest and the user is able to read the information and to give his feedback about the place in a four levels scale. Taking into consideration the places the users interacted to, and their opinions on each place, the system also provides the users with recommendations of other places of interest. A local 
database (SQLite) allows some operations when the Internet connection is not available.

The current version of our prototype only supports interactions through qr-codes. However, future versions of the system should provide support for NFC interactions. This is important because NFC interactions can be more spontaneous and less intrusive to the users.

\section{Prototype and experiments}

Although the system has not yet been fully implemented, first experiments show that the system can help the tourists to interact with places of interest, helping them in their visits and also to promote some shops and services. So far the prototype supports interactions with qr-codes installed in the places of interests and it presents information associated to the place. It also allows the user to search for places of interest and to search for places of interest that are similar to the place he has interacted to. The system recommends to the user a list of places of interest which are similar to the ones he previously interact to, or similar to the ones the user gave positive feedback.

To evaluate some features of the system, a set of places of interest were inserted in the system (10 places of interest organized within 3 categories. Religious: 4 places of interest; nature and gardens: 3 places of interest; historic places: 3 places of interest. 4 of them are classified within two categories). Below some functionalities of the system are described.

Fig. 2a shows the main screen presenting the four main functionalities to the user: search for places of interest; recommend places of interest according to the user location; recommending pedestrian tours and interacting with a place of interest.

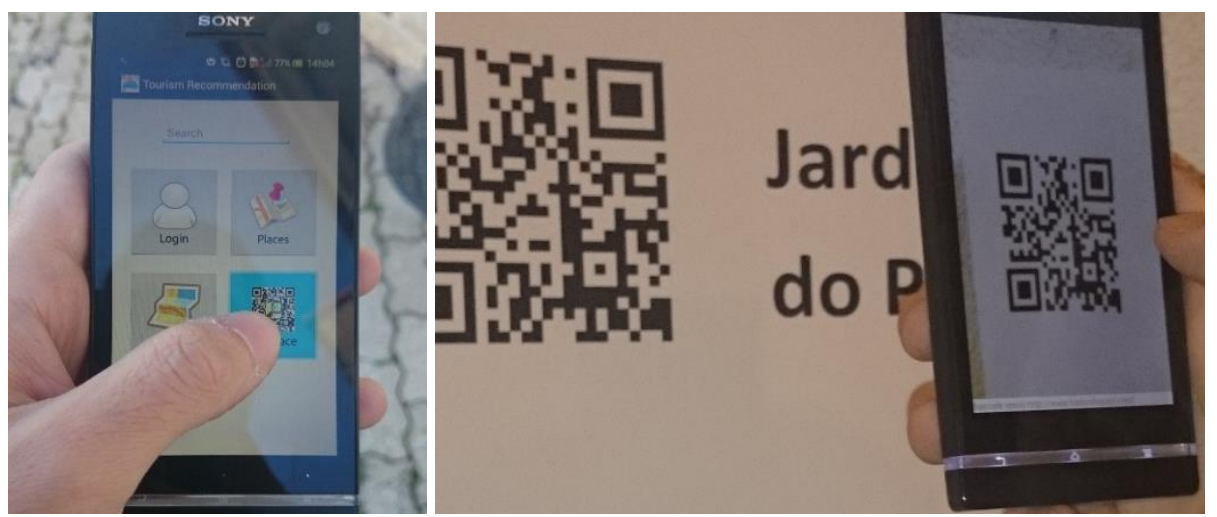

Fig. 2. a) Main screen. b) Interaction with a place of interest.

When a user interacts with a place of interest using his smartphone camera to read the qr-code (Fig. 2b) a new screen with information about the place is presented (see Fig. 3a).

Recommendations can be obtained from two distinct screens. The first one is from the main menu, selecting the places functionality. In this case a set of places of interest 
are displayed according to the user location. The second one, within the screen related to a specific place of interest (see Fig. 3a), the user selects the option recommend other places and a new screen with a list of places is presented (see Fig. 3b). This list is sorted according to the appropriateness to the user' interests and distance from the user location. When there are places of interest that are promoted by the system (e.g. shops, services) they are presented differently from the others places and they contain a statement indicating they are commercial places related to the interests of the tourist.
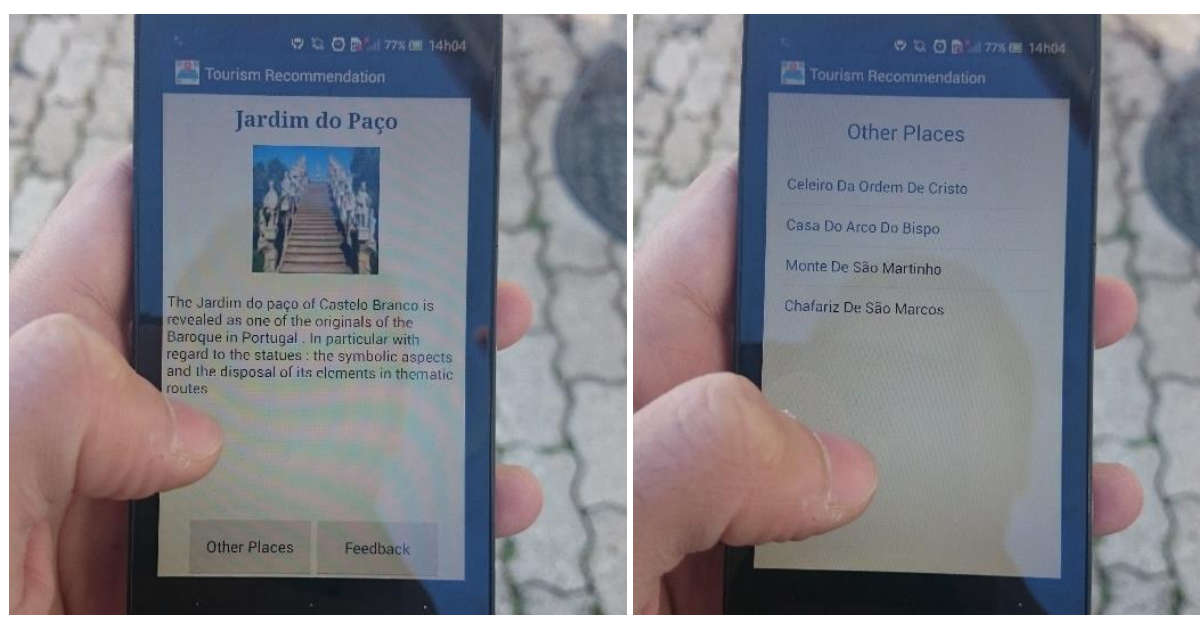

Fig. 3. a) Information about a specific place of interest. b) Recommended places of interest.

Within the screen related to a place of interest the user is able to get recommendations about new places of interest and he is also able to give his opinion about the place (four levels scale). This feedback is central to improve the recommendations accuracy.

At this time the pedestrian tour recommendation functionality only provides the user with the route between the user location and the first place of interest recommended to him.

\section{Conclusions and future work}

In this work we describe a system to support the promotion of tourism resources and services by recommending pedestrian tours and places of interest in accordance with the interests of the visitors. The system explores information about user' interactions with places of interest, performed with their mobile phones and their opinions about each place, to recommend new places of interest and pedestrian tours and to promote product and services that are related to their interests.

Initial experiments show that interaction mechanism is intuitive and simple to use and that the system provides recommendations according to the location and the interests of the users. However, the system has not yet been realized with a complete instance and it does not fully comply with our system design. As a consequence, our 
plan for the future work is to develop a full functionality prototype. This includes: 1) improve the recommendation process, including recommendations based on the preferences of similar users; 2) improve pedestrian tours recommendation and navigation; 3) perform more experiments with different users and different types of scenarios to get more information about the recommendation accuracy and about the users' participation and acceptance.

\section{References}

1. Ludwig, B., B. Zenker, and J. Schrader, Recommendation of Personalized Routes with Public Transport Connections, in Intelligent Interactive Assistance and Mobile Multimedia Computing, D. Tavangarian, et al., Editors. 2009, Springer Berlin Heidelberg. p. 97-107.

2. Wan, Z. Personalized Tourism Information System in Mobile Commerce. in Management of e-Commerce and e-Government, 2009. ICMECG '09. International Conference on. 2009.

3. Zhao, Y.-L., et al., Personalized Recommendations of Locally Interesting Venues to Tourists via Cross-Region Community Matching. ACM Trans. Intell. Syst. Technol., 2014. 5(3): p. 126.

4. Baltrunas, L., et al., Context-Aware Places of Interest Recommendations for Mobile Users, in Design, User Experience, and Usability. Theory, Methods, Tools and Practice, A. Marcus, Editor. 2011, Springer Berlin Heidelberg. p. 531-540.

5. Lucchese, C., et al., How Random Walks Can Help Tourism, in Advances in Information Retrieval, R. Baeza-Yates, et al., Editors. 2012, Springer Berlin Heidelberg. p. 195-206.

6. Hao, Q., et al., Equip tourists with knowledge mined from travelogues, in Proceedings of the 19th international conference on World wide web2010, ACM: Raleigh, North Carolina, USA. p. 401-410.

7. Adomavicius, G. and A. Tuzhilin, Toward the Next Generation of Recommender Systems: A Survey of the State-of-the-Art and Possible Extensions, in IEEE Transactions on Knowledge and Data Engineering2005.

8. Pazzani, M.J. and D. Billsus, Content-based recommendation systems, in The adaptive web, B. Peter, K. Alfred, and N. Wolfgang, Editors. 2007, Springer-Verlag. p. 325-341.

9. Das, A.S., et al. Google news personalization: scalable online collaborative filtering. in 16th international conference on World Wide Web. 2007. Banff, Alberta, Canada

10. Campos, L.M.d., et al., Combining content-based and collaborative recommendations: A hybrid approach based on Bayesian networks. Int. J. Approx. Reasoning, 2010. 51(7): p. 785-799. 